\title{
QUALIDADE DE VIDA DE PACIENTES APÓS A REALIZAÇÃO DE CIRURGIA BARIÁTRICA
}

\section{QUALITY OF LIFE OF PATIENTS AFTER BARIATRIC SURGERY}

Fernanda Costa Batista

Lucyana Silva Luz

Kedilla Dias Souza

Amélia Cristina Stival Duarte

\section{INTRODUÇÃO}

A obesidade é uma doença crônica relacionada ao acúmulo excessivo de gordura corporal, causada pela interação de aspectos multifatoriais como hábitos de vida, carga genética e fatores emocionais ${ }^{1}$. Atualmente a obesidade apresenta alta morbidade e mortalidade com aproximadamente 2,8 milhões de mortes anuais². Acompanhando o crescimento da obesidade na população brasileira, o número de cirurgias bariátricas elevou-se consideravelmente nos últimos anos, com a realização de mais de 100 mil cirurgias somente no ano de 2017, representando5,6\% a mais do que em 2016. O Brasil é tido como o segundo país do mundo em número de cirurgias bariátricas realizadas ${ }^{3}$, uma vez que, este método é considerado o de maior eficiência a longo e a curto prazo, já que promove redução de peso, melhora da qualidade de vida e das comorbidades ${ }^{4}$.

No Brasil, as mudanças demográficas e epidemiológicas dos últimos anos tornaram as Doenças Crônicas Não Transmissíveis mais prevalentes do que os agravos infecciosos, revelando o sobrepeso e a obesidade como importantes fatores de risco para acometimento de outras morbidades ${ }^{5}$. Dados do Vigitel 2016 revelam crescimento da obesidade em $60 \%$ nos últimos 10 anos, com prevalência de 18,9\% de indivíduos obesos nas capitais brasileiras e Distrito Federal no ano supracitado 4 . 
Estimativas para 2020 apontam cerca de cinco milhões de óbitos atribuídos ao excesso de peso ${ }^{6}$.

Dentre os métodos disponíveis de avaliação de peso, o Índice de Massa Corporal (IMC) é o instrumento mais utilizado para avaliação de adiposidade física e grau de corpulência em um indivíduo, tornando-se a principal ferramenta adotada pela Organização Mundial de Saúde (OMS) para a identificação de peso adequado e associação de risco com outras doenças crônicas ${ }^{1,7}$. Classifica-se como obeso o indivíduo com IMC superior a $30 \mathrm{~kg} / \mathrm{m}^{2}$, sendo possível ainda segmentar a obesidade de acordo com pontos de corte baseados no IMC, por meio das especificações: grau I, quando o valor de IMC for de $30-34,9 \mathrm{~kg} / \mathrm{m}^{2}$; grau II, quando o valor de IMC for de $35-39,9 \mathrm{~kg} / \mathrm{m}^{2}$; e grau III, quando o valor de IMC for superior a $40 \mathrm{~kg} / \mathrm{m}^{27}$.

O tratamento da obesidade consiste em uma série de abordagens, dentre elas a mudança de hábitos de vida, reeducação alimentar, prática de atividade física, redução de peso, associado ou não a tratamento farmacológico, e a cirurgia bariátrica ${ }^{8}$. Contudo, Mancini ${ }^{1}$ refere que os métodos não invasivos de tratamento da obesidade apresentam grande índice de recidiva.

A obesidade está associada à incapacidade funcional e maior mortalidade, apresentando diversas doenças e problemas relacionados nos vários sistemas e aparelhos corporais, dentre eles, sistema respiratório, sistema digestório, sistema nervoso, pele, função sexual e reprodutora e função psicossocial, além de estar associada a outras comorbidades, tais como aumento do risco cirúrgico e anestésico e propensão a acidentes ${ }^{1}$.

Segundo a Sociedade Brasileira de Cirurgia Bariátrica e Metabólica (SBCBM), os critérios de elegibilidade para indicação de gastroplastia redutora, cirurgia bariátrica, incluem pessoas portadoras de obesidade mórbida que tenham IMC superior a $40 \mathrm{~kg} / \mathrm{m}^{2}$, sem sucesso no tratamento clínico longitudinal, que inclui orientação e apoio para mudança de hábitos, realização de dieta, atenção psicológica, prescrição de atividade física e, se necessário, farmacoterapia, realizado na Atenção Básica e/ ou Atenção Ambulatorial Especializada por no mínimo dois anos e que tenham seguido protocolos clínicos; ou ainda com IMC entre $35-39,9 \mathrm{~kg} / \mathrm{m}^{2}$ quando associado a comorbidades, como, alto risco cardiovascular, diabetes mellitus, hipertensão arterial, doenças articulares degenerativas e apneia do sono ${ }^{1,9}$. Dentre os 
benefícios resultantes da cirurgia bariátrica, destaca-se a redução do peso corporal, a melhora das comorbidades relacionadas a obesidade e a melhora do estado psicossocial e da qualidade de vida?

De acordo com Major ${ }^{10}$, a qualidade de vida de pessoas obesas é inferior à de pessoas com peso corporal adequado, resultante do comprometimento das funções físicas, mentais e sociais provocadas pelo excesso de adiposidade corporal, contudo a intervenção cirúrgica pode promover melhora do estado de saúde geral e bem-estar psíquico e emocional do indivíduo obeso. Resultados do estudo desenvolvido por esse autor, demonstraram considerável redução de massa corporal em todos os pacientes após 1 ano da realização da cirurgia bariátrica, com minoração significativa da gravidade das comorbidades ${ }^{10}$. A partir de aplicação do questionário de qualidade de vida, formulário SF-36, foi identificada uma qualidade de vida reduzida antes do tratamento cirúrgico, com aumento significativo 12 meses após a cirurgia, que pode ser verificado por meio do índice de qualidade de vida que elevou-se de 85,2 para 145,1 pontos neste mesmo questionário ${ }^{10}$.

A qualidade de vida é um termo abrangente, à medida que pode ser utilizado a partir de dois aspectos: na linguagem cotidiana da população em geral, sintetizando seu significado em melhorias ou alto padrão de bem estar na vida e no contexto da pesquisa científica, em diferentes campos do saber, no qual sua definição se encontra em construção, sendo analisada a partir da subjetividade e multidimensionalidade ${ }^{11,12}$.

De acordo com Minayo ${ }^{13}$, qualidade de vida de um indivíduo está associada ao grau de satisfação vivenciado no contexto familiar, amoroso, social, ambiental e à sua estética existencial, sendo considerada uma construção social a partir da relatividade cultural. A Organização Mundial de Saúde apresenta a seguinte definição para o vocábulo: é "a percepção do indivíduo de sua inserção na vida no contexto da cultura e sistemas de valores nos quais ele vive e em relação aos seus objetivos, expectativas, padrões e preocupações"11,12.

A avaliação dos resultados da cirurgia bariátrica no paciente obeso deve utilizar alguns parâmetros essenciais, tais como a perda de peso, a melhoria das comorbidades relacionadas ao excesso de peso e as mudanças nos indicadores de qualidade de vida ${ }^{14}$. Para esse fim, utiliza-se o Bariatric Analysis and Reporting Outcome System (BAROS), um protocolo específico de avaliação das mudanças 
advindas na vida do paciente após intervenção cirúrgica na obesidade ${ }^{14}$. A utilização do protocolo BAROS possibilita análise, uniformização e comparação dos resultados da cirurgia bariátrica, obtidos por diferentes profissionais, em grupos de paciente no Brasil e no mundo, através de sua metodologia global ${ }^{15,16}$.

A evolução e complicações da obesidade suscitam a ocorrência de variados problemas aos pacientes obesos, nos seus aspectos de saúde geral, psicológicos e sociais, os quais resultam em déficit de qualidade de vida e alterações na sua dinâmica fisiológica e funcional, resultando nos seguintes danos: prejuízo em mobilidade e prática de exercícios físicos, dores articulares, falta de confiança pessoal e baixo estima, aumento do estresse, diminuição do humor e transtornos depressivos ${ }^{17}$. Diante de todas as complicações geradas pela obesidade e pelas mudanças negativas de hábito de vida imposta por este agravo, supõe-se que a cirurgia bariátrica proporcione benefícios aos pacientes, tais como perda de peso, remissão de comorbidades e melhora da qualidade de vida em seus diversos aspectos (psicológico, físico, social, familiar, afetivo dentre outros).

O presente estudo propõe-se a buscar resposta para os seguintes questionamentos: Ocorre melhora na qualidade de vida do paciente obeso após realização da cirurgia bariátrica? Qual o nível de satisfação desse paciente com os resultados obtidos pós procedimento cirúrgico? Quais as mudanças verificadas no estilo de vida do paciente?

Assim, este estudo, objetivou caracterizar a qualidade de vida dos pacientes submetidos à cirurgia bariátrica do Programa de Controle e Cirurgia da Obesidade (PCCO) de um hospital de grande porte na cidade de Goiânia - GO.

\section{METODOLOGIA}

Trata-se de estudo clínico, descritivo, de abordagem quantitativa que investigou as mudanças na qualidade de vida de pacientes após a realização de cirurgia bariátrica. A presente pesquisa foi realizada em um hospital de grande porte de Goiânia, especializado em média e alta complexidade que oferta serviços vinculados ao Sistema Único de Saúde (SUS). Dentre os programas ofertados pelo hospital, destaca-se como alvo de estudo desta pesquisa, o Programa de Controle e Cirurgia 
da Obesidade (PCCO), no qual atende aproximadamente 1100 pacientes em pré e pós-operatório de cirurgia bariátrica.

A coleta de dados foi realizada pela enfermeira residente e pesquisadora responsável, no período de agosto a dezembro de 2018 (5 meses), com pacientes após 6 meses de realização da cirurgia bariátrica. Este período foi estabelecido após pesquisa na literatura no intuito de abordar os pacientes quando estes começam a praticar atividade física, iniciaram uma alimentação diferenciada, e poder vivenciar as mudanças na rotina diária

Foram incluídos na pesquisa participantes do PCCO que tinham idade igual ou superior a 18 anos, de ambos os gêneros, que realizaram cirurgia bariátrica, no mínimo há 6 meses e que assinaram o Termo de Consentimento Livre e Esclarecido (TCLE).

A amostra obtida foi de 34 participantes que estavam em pós-operatório de cirurgia Bariátrica.

Para coleta dos dados foi utilizada o Protocolo Bariatric Analisys and Reporting Outcome System (BAROS) (Anexos I e II), que consiste na obtenção de informações do paciente após a cirurgia bariátrica abordando três domínios de interesse: redução do peso, melhora de comorbidades e mudança na qualidade de vida.

Os dados sobre perda de peso foram obtidos diretamente com o paciente e através de informações obtidas em prontuário e posteriormente analisados de acordo com tabela oriunda do protocolo BAROS. A tabela segue sistema de pontuação numérica, variando de 0 a 3 pontos em conformidade com a porcentagem de perda de peso. Caso haja relato de aumento de peso em relação a período anterior a cirurgia deverá haver a dedução de 1 ponto na pontuação final.

As mudanças relacionadas à condições médicas após a realização da cirurgia bariátrica foram coletadas em prontuário, bem como as principais comorbidades do paciente anteriormente à intervenção cirúrgica. A pontuação sobre o prognóstico dessas comorbidades após a realização da gastroplastia também foi obtida a partir do protocolo BAROS. A presença de complicações e reoperações no pós operatório deduz pontos na somatória final.

As comorbidades associadas à obesidade que foram consideras para a presente pesquisa foram as relacionadas pela Associação Brasileira para o Estudo da 
Obesidade e da Síndrome Metabólica: síndrome metabólica (caracterizada pela associação entre obesidade e elevação da pressão arterial, da glicemia em jejum e do triglicérides com redução de níveis de HDL); Diabetes Mellitus tipo 2; Doença cardiovascular (infarto do miocárdio, angina, insuficiência cardíaca congestiva, acidente vascular cerebral, hipertensão e fibrilação atrial); apneia obstrutiva do sono; depressão; neoplasias; osteoartrose ${ }^{7}$.

Os dados sobre qualidade de vida foram obtidos pelo Questionário de Qualidade de vida de Moorehead-Ardelt (QoL)(ANEXO II), um instrumento integrante do protocolos BAROS que permitiu analisar as repercussões da cirurgia bariátrica na qualidade de vida do indivíduo obeso por meio de cinco dimensões: autoestima, disposição para atividade física, relacionamento social, disposição para o trabalho e interesse sexual ${ }^{18}$. O questionário possui perguntas concernentes às áreas citadas anteriormente, no qual cada alternativa corresponde a uma pontuação, gerando escore final no questionário. A somatória final dos pontos obtidos nos três parâmetros permitiu a classificação dos resultados em sucesso ou falha da intervenção cirúrgica e categorizou grupos de acordo com os resultados ${ }^{15}$.

Para obtenção de dados antropométricos, demográficos e de comorbidade foi aplicado ficha de informações individuais (Apêndice B) e coleta por análise de prontuário do paciente.

Esta pesquisa passou pelo Comitê de Ética em Pesquisa (CEP) do hospital em questão e iniciou após sua aprovação e emissão de Parecer Consubstanciado, respeitando as Diretrizes e Normas de Pesquisa em Seres Humanos brasileiros, como a Resolução CNS 466/2012 do Conselho Nacional de Saúde -CNS. CAAE: 83327818.1.0000.0035.

A análise estatística foi desenvolvida no programa IBM SPSS Statistics for Windows 16.0. As variáveis contínuas foram expressas em médias, com seus respectivos desvios padrões; as variáveis categóricas foram apresentadas por meio das frequências absolutas e relativas.

Para testar as associações entre as variáveis e médias entre grupos foram utilizados testes paramétricos (testT para amostras pareadas e teste T de Student) e não paramétricos (Teste de Wilcoxone teste de Mann-Withney). Para todas as 
análises, foi considerado um nível de significância de 5\% (valor $p<0,05)$ e intervalo de confiança de $95 \%$.

\section{RESULTADOS}

A amostra foi composta por 34 pacientes em período médio de pós-operatório de 10,6 meses $(D P=4)$ de cirurgia bariátrica pela técnica de Bypass Gástrico em $Y$ de Roux, com média de idade de 40,5 anos ( $\mathrm{DP}=11,1)$,

O perfil socioeconônimo dos pacientes avaliados foram distribuídos por sexo, estado civil e escolaridade, como ilustrado na tabela 1. Foi possível verificar que a maioria dos pacientes era do sexo feminino (94,1\%).

Tabela 1.Perfil socioeconômico da amostra investigada

\section{Variável}

\section{Frequência}

$$
\text { n (34) } \%
$$

\section{Sexo}

Feminino

32

94,1

Masculino

2

5,9

\section{Estado civil}

Casado(a)

Solteiro(a)

Divorciado(a)

União estável

2

5,9

\section{Escolaridade}

Ensino Fundamental incompleto

Ensino Fundamental completo

Ensino Médio incompleto

Ensino Médio completo 10 29,4

Ensino Superior incompleto $7 \quad 20,6$

Ensino Superior completo 1

2,9

As médias de IMC e peso, pré e pós operatórios, estão na tabela 2. 
Tabela 2. Evolução do peso e do Índice de Massa Corporal após a realização da cirurgia bariátrica

\begin{tabular}{lcccc}
\hline \multirow{2}{*}{ Variável } & Antes da cirurgia & \multicolumn{1}{c}{ Atual } & \multirow{2}{*}{ IC $^{*}$} & \multirow{2}{*}{ Valor $\mathbf{p}^{\star *}$} \\
\cline { 2 - 3 } & Média \pm DP & Média \pm DP & & \\
\hline IMC $\left(\mathrm{kg} / \mathrm{m}^{2}\right)$ & $49,49 \pm 7,19$ & $34,18 \pm 6,50$ & $13,87-$ & $<0,001$ \\
Peso $(\mathrm{kg})$ & $128,5 \pm 24,02$ & $89,2 \pm 20,7$ & $35,5-43,1$ & $<0,001$
\end{tabular}

${ }^{*}$ IC: intervalo de confiança; ${ }^{* *}$ teste T para amostras pareadas

$\mathrm{Na}$ avaliação das comorbidades pré operatórias, a pesquisa mostrou que $67,6 \%$ (n: 23) da amostra apresentam algum tipo de comorbidade, destes, aproximadamente 40\% (n:9) apresentam apenas uma comorbidade e 60\% (n:14) apresentam duas ou mais comorbidades associadas, sendo as mais encontradas, hipertensão arterial sistêmica(HAS), com $55,8 \%$ e diabetes mellitus (DM), com $32,4 \%$ da amostra.

Em relação a ocorrência de complicações pós operatórias maiores e menores, $52,9 \%$ dos pacientes apresentaram algum tipo de complicação, como hérnia incisional $(20,8 \%)$, queda de cabelo $(20,8 \%)$, seroma $(16,7 \%)$ e reabordagem cirúrgica $(4,2 \% \%)$.

A avaliação da qualidade de vida ocorreu por meio do instrumento BAROS, com cinco domínios: autoestima, disposição para atividade física, interação social, capacidade de trabalho, e interesse sexual, como ilustrado na tabela 3.

Tabela 3. Classificação do questionário sobre qualidade de vida Moorehead-Ardelt de acordo com as questões avaliadas.

\section{Avaliação}

Muito $\quad$ Muito

Questão Avaliada menos Menos mesmo Mais mais

Como se sente comparando a época

anterior ao tratamento

$6 \quad 28$

(autoestima)

$0(0 \%) \quad 0(0 \%) \quad 0(0 \%) \quad(17,6 \%)$

$(82,4 \%)$ 
Capacidade de participar de atividades físicas

Relacionamento social

Trabalho

Interesse por sexo

$$
0(0 \%)
$$

$0(0 \%)$ 2

$0(0 \%)$

$(5,8 \%)$
3

15

$(8,8 \%) \quad(44,1 \%) \quad 16(47 \%)$

9

7

\section{DISCUSSÃO}

A maioria dos pacientes avaliados foram do sexo feminino, corroborando com os estudos de Maia $^{19}$ e Castanha ${ }^{20}$, que apresentaram uma taxa superior do sexo feminino, de $84 \%$ e $89,3 \%$ respectivamente. De acordo com o estudo de Carvalho ${ }^{21}$, esse fato pode ser explicado pela maior preocupação das mulheres em relação a aparência, já os homens procuram o tratamento cirúrgico apenas quando a obesidade começa a afetar suas atividades físicas diárias.

Os níveis de escolaridade mais freqüentes foram ensino médio completo e ensino fundamental completo, dados similares foram encontrados no estudo de Maia ${ }^{19}$, que avaliou o perfil nutricional e a qualidade de vida de 56 pacientes após a realização da cirurgia bariátrica.

A média de peso e IMC encontrados no período pré- operatório foi de 128,5 $\mathrm{kg} \pm 24,02$ e 49,49kg $\pm 7,19$, respectivamente. No período pós operatório a média do peso e IMC foi para 89,2 $\mathrm{kg} \pm 20,7$ e 34,18 $\pm 6,50$, respectivamente. Tanto os dados pré como os pós operatórios corroboram com os estudos de Castanha ${ }^{20}$ e Maia ${ }^{19}$, nestes estudos os dados encontrados também mostram redução dos valores de IMC. No estudo de Castanha ${ }^{20}$, o IMC variou de 48, 1 no pré operatório para 31,05 no pós operatório, no estudo de Maia ${ }^{19} \mathrm{O}$ IMC foi de 50,1 para 35,5.

Ao analisar a perda de peso com a cirurgia bariátrica, foi observado que na presente pesquisa, assim como em outros estudos, o procedimento vem se mostrando eficaz, já que houve melhora significativa do quadro de obesidade, passando da média de obesidade grau III para grau I. A diminuição do IMC resulta em expressiva redução 
na mortalidade cardiovascular e na mortalidade por todas as causas associadas ao excesso de peso ${ }^{20}$.

No estudo de Maia ${ }^{19}$, as comorbidades mais frequentes foram HAS (84\%) e DM tipo $2(64 \%)$, reafirmando que a obesidade é fator de risco para desenvolvimento de doenças crônicas ${ }^{20}$.

O percentual de melhora das comorbidades após a realização da cirurgia foi importante e apresentou diferença significativa $(p<0,001)$, dos pacientes que apresentavam pelo menos HAS, 94,1\% referiram melhora da doença, em relação aos que apresentavam pelo menos DM, 97,1\% referiram melhora do quadro. No estudo de Carvalho ${ }^{21}$ sobre qualidade de vida de pacientes submetidos a cirurgia bariátrica, todos os 46 pacientes entrevistados relataram melhora das comorbidades após perda de peso. Outros estudos também mostram a eficácia da cirurgia bariátrica nas diversas comorbidades associadas à obesidade 17,19,20,21.

Castanha ${ }^{20}$ questiona se o percentual de melhora do diabetes mellitus tipo II se manteria elevado caso a pesquisa abordasse períodos pós-cirúrgicos mais prolongados.

No estudo de Barros ${ }^{17}$, sobre avaliação dos resultados após realização da cirugia bariátrica, foram entrevistados 92 pacientes, as complicações mais referidas foram queda de cabelo $(74,2 \%)$ e hérnia incisional $(30,6 \%)$. No estudo de Castanha ${ }^{20}$, as complicações mais frequentes observadas na amostra estudada foram queda de cabelo $(79,6 \%)$, deficiência nutricional $(37,9 \%)$ e anemia (35\%).

Dados semelhantes ao questionário de qualidade de vida aplicado neste estudo, foram encontrados no estudo de Maia $^{19}$, em que $73 \%$ da amostra avaliou a qualidade de vida como muito melhorada e $27 \%$ como melhorada. Em estudo consoante com 103 participantes, 94,2\% consideram que a autoestima está melhor ou muito melhor, assim como 90,3\% aumentaram a frequência de suas atividades físicas, $55,3 \%$ melhoraram seus relacionamentos sociais, $79,6 \%$ sentem-se mais capazes de trabalhar e $51,4 \%$ avaliaram que o interesse por sexo está melhor ou muito melhor, corroborando os achados da literatura ${ }^{20}$.

Em um estudo sobre análise da qualidade de vida de pacientes submetidos à cirurgia bariátrica e hábitos de atividade física pós-cirurgia, quando analisado o 
questionário Baros, $90 \%$ dos pacientes descreveram se sentirem muito melhor de forma geral após a realização da cirurgia bariátrica ${ }^{22}$.

O BAROS é considerado o modo mais eficaz de avaliação global do tratamento cirúrgico da obesidade ${ }^{23}$, contudo devido as limitações do serviço de saúde analisado, como falta de padronização do registro de complicações no período pós-operatório e reavaliações das comorbidades, dificultou a análise da ficha de pontuação das comorbidades e complicações.

\section{CONCLUSÃO}

Os resultados do estudo demonstraram que a realização da cirurgia bariátrica está associada a uma alta perda de peso, sendo um procedimento eficaz no tratamento da obesidade mórbida, no controle das comorbidades e na melhora da qualidade de vida de forma geral. Porém, salienta-se a importância de avaliar a eficácia da cirurgia com maior tempo de pós-operatório, visando observar se haverá manutenção, perda ou reganho de peso.

Foi evidenciado também a ocorrência de complicações pós cirúrgicas, por isso a importância de sempre orientar os pacientes quanto aos riscos e benefícios da realização da cirurgia bariátrica, bem como assegurar o acompanhante perioperatório com qualidade.

\section{REFERÊNCIAS}

1. Mancini MC. Tratado de obesidade. $2^{\mathrm{a}}$ ed. Rio de janeiro: Guanabara Koogan; 2015. p. 782.

2. Word health organization. 10 facts on obesity. Genebra; 2017. Available from: https://www.who.int/features/factfiles/obesity/en/.

3. Sociedade Brasileira de Cirurgia Bariátrica e Metabólica. Número de cirurgias bariátricas no Brasil aumenta 46,7\%. 2018. Disponível em: https://www.sbcbm.org.br/numero-de-cirurgias-bariatricas-no-brasil-aumenta467/. 
4. Brasil. Ministério da saúde. Vigitel Brasil 2016: Vigilância de fatores de risco e proteção para doenças crônicas por inquérito telefônico. Brasília; 2017.

5. Brasil. Ministério da Saúde. Perspectivas e desafios no cuidado às pessoas com obesidade no SUS: resultados do Laboratório de Inovação no manejo da obesidade nas Redes de Atenção à Saúde. Brasília; 2014.

6. Malta, DC, Andrade SC, Claro RM, Bernalrti, Monteiro CA. Evolução anual da prevalência de excesso de peso e obesidade em adultos nas capitais dos 26 estados brasileiros e no Distrito Federal entre 2006 e 2012. Rev. bras. epidemiol. 2014; 17(Suppl 1): 267-276.

7. Associação Brasileira para o Estudo da Obesidade e da Síndrome Metabólica (ABESO). Diretrizes brasileiras de obesidade. São Paulo; 2016.

8. Rossum JFV, Nakaoka VY, Rodrigues RO, Assunção RDL. Uma abordagem atual da obesidade. Brazilian Journal of Surgery and Clinical Research. 2014;9(1):54-59.

9. Comissão Nacional de Incorporação de Tecnologias no SUS (CONITEC). Cirurgia bariátrica por laparoscopia - Relatório de recomendação. Brasília; 2017.

10. Major $P$, Matłok $M$, Pędziwiatr $M$, Migaczewski $M$, Budzyński $P$, Stanek $M$ et al. Quality of Life After Bariatric Surgery. Obes Surg. 2015;25(9):1703-10.

11. Almeida MAB, Gutierrez GL, Marques, R. Qualidade de vida: definição, conceitos e interfaces com outras áreas de pesquisa. Escola de Artes, Ciências e Humanidades - EACH/USP. 2012. p 142.

12. Seidl EMF, Zannon CMLC. Qualidade de vida e saúde: aspectos conceituais e metodológicos. Cad. Saúde Pública. 2004; 20(2): 580-588.

13. Minayo MCS, Hartz ZMA, Buss PM. Qualidade de Vida e saúde: um debate necessário. Ciênc. saúde coletiva. 2000; 5(1): 7-18.

14. Oria HE, Moorehead MK. Bariatric analysis and reporting outcome system (BAROS). Obes Surg. 1998; 8(5): 487-499.

15. Pinto RD. Avaliação da qualidade de vida através do questionáio B.A.R.O.S (Bariatric Analisys and Reporting Outcome System) dos pacientes submetidos a derivação bileo-pancreática [dissertação]. Rio Grande do Sul: Universidade Federal do Rio Grande do sul; 2000. 
16. Barros LM, Moreira RAN, Frota NM, Caetano JA. Mudanças na qualidade de vida após a cirurgia bariátrica. Rev enferm UFPE. 2013; 7(5): 1265-75.

17. Barros LM, Frota NM, Moreira RAN, Araújo TM, Caetano JA. Avaliação dos resultados da cirurgia bariátrica. Rev Gaúcha Enferm. 2015; 36(1): 21-27.

18. Chaves LCL, Carvalho AH, Almeida HG, Chaves IKL, Neves MW. Qualidade de vida de pacientes submetidos à cirurgia bariátrica, por meio da aplicação do questionário BAROS. Rev. para. Med. 2012; 26(3).

19. Maia RP, Silva PCC, Duarte ACS, Costa RM. Avaliação do perfil nutricional e qualidade de vida após realização de cirurgia bariátrica em um Hospital Público de Goiânia. Demetra. 2018; 13(1):147-164.

20. Castanha CR, Ferraz AAB, Castanha AR, Belo GQMB, Lacerda RMR, Vilar L. Avaliação da qualidade de vida, perda de peso e comorbidades de pacientes submetidos à cirurgia bariátrica. Rev Col Bras Cir. 2018; 45(3):1864.

21. Carvalho LA, Pires RCCP, Rebelo TJ, Silva L. Cirurgia bariátrica no Hospital Santa Casa de Misericórdia de Belo Horizonte/MG. Revista da Universidade Vale do Rio Verde. 2013;11(1):195-205.

22. Segura DCA, Nascimento FC, Beuren TR, Wozniak SD. Análise da qualidade de vida de pacientes submetidos à cirurgia bariátrica e hábitos de atividade física pós-cirurgia. Braz. J. Surg. Clin. Res. 2014 ;9(1):12-19.

Nicareta JR, Freitas ACT, Nicareta SM, Nicareta C, Campos ACL, Nassif PAN et al. Análise crítica do método BAROS. ABCD, arq. bras. cir. dig. 2015; 28( Suppl 1 ): 73-78. 


\section{APÊNDICE A}

\section{TERMO DE CONSENTIMENTO LIVRE E ESCLARECIDO - TCLE}

\section{Caro participante,}

Você está sendo convidado(a) para participar, como voluntário(a), de uma pesquisa. Após ser esclarecido(a) sobre as informações a seguir e no caso de aceitar fazer parte do estudo, assine ao final deste documento que estará em duas vias, uma delas é sua e a outra é nossa, das pesquisadoras. Em caso de dúvida sobre a pesquisa você poderá entrar em contato com a pesquisadora responsável, Fernanda Costa Batista e a tutora de enfermagem Lucyana da Silva Luz pelos respectivos números telefônicos: (062) 984272584 e (062) 98651-7693.

Se você tiver alguma dúvida sobre os seus direitos como participante nesta pesquisa ou questões éticas, você poderá entrar em contato com o Comitê de Ética em Pesquisa do Hospital Alberto Rassi - HGG (um grupo não remunerado, formado por diferentes profissionais e membros da sociedade, que avaliam um estudo para julgar se ele é ético e garantir a proteção dos participantes), situado na Avenida Anhanguera, ㄲo 6.479, Setor Oeste, Goiânia, Goiás, pelo telefone (62) 3209-9917, de segunda a sexta-feira, no horário de $07 \mathrm{~h}$ às $18 \mathrm{~h}$ ou pelo e-mail: hgg.cep@idtech.org.br.

\section{Informações sobre a pesquisa:}

O presente projeto de pesquisa tem como título: "Qualidade de Vida de Pacientes após realização de Cirurgia bariátrica”. Assim, esta pesquisa tem como objetivo geral: Caracterizar a qualidade de vida dos pacientes submetidos à cirurgia bariátrica do Programa de Controle e Cirurgia da Obesidade (PCCO) de um hospital de grande porte na cidade de Goiânia - GO.

A coleta de dados será por meio de um questionário estruturado e as informações obtidas serão utilizadas apenas para fins científicos, as quais serão 
analisadas e apresentadas publicamente, independentes dos seus resultados. No sentido de preservar a sua privacidade durante a coleta de dados, o preenchimento da ficha de dados complementares e do questionário será feita em local reservado (consultório do Ambulatório de Medicina Avançada). Assim como também garantimos a você que será mantida a confidencialidade das suas informações, sendo restrito o acesso somente a nós, pesquisadores deste estudo.

Ressalta-se que a sua participação não the beneficiará diretamente, porém contribuirá no crescimento profissional da categoria de enfermagem e dos conhecimentos específicos nesta área. Ademais, será favorecida indiretamente, ao receber a assistência de profissionais mais bem preparados para a intervenção na realidade.

Sua participação consiste em responder perguntas de um questionário conforme o roteiro apresentado no momento da coleta dos dados acrescido da ficha de informações individuais. Lembramos que a sua participação é voluntária e a qualquer momento você pode recusar-se a responder qualquer pergunta, ou desistir de participar, retirando assim o seu consentimento. Sua recusa não trará nenhum prejuízo para sua assistência no hospital e nem para a instituição. Também será garantido a você o direito de solicitar informações, acompanhar e ter acesso ao desenvolvimento da pesquisa por meio de relatórios parciais da mesma junto às pesquisadoras.

Você receberá uma cópia deste termo no qual consta o e-mail da pesquisadora responsável, facilitando assim, o acesso à mesma para esclarecimento de suas dúvidas quanto à pesquisa e sua participação. Informamos ainda, que não haverá nenhum tipo de pagamento ou gratificação financeira. Porém, está previsto que você terá direito ao ressarcimento, pelo pesquisador, de despesas gastas com a pesquisa (alimentação, transporte e outros). Você terá ainda direito a buscar indenização por parte dos pesquisadores, caso se sinta prejudicado por participar deste estudo. Entretanto a decisão final sobre a indenização ficará a cargo da justiça comum.

Como forma de garantir o sigilo e anonimato de sua identificação e da instituição todos os documentos e informações (sejam físicos ou digitados), obtidos na coleta de dados permanecerão sob a guarda da pesquisadora responsável, por um período de cinco (5) anos após o término da pesquisa, sendo destruídos antes de 
serem descartados, conforme as recomendações da Resolução CNS 466/2012do Conselho Nacional de Saúde (BRASIL, 2012).

Oportunamente informamos a você que, ao final desta pesquisa a mesma será apresentada em eventos científicos e, publicada em periódico de circulação nacional, independentemente dos seus resultados, como forma de dar visibilidade ao tema estudado.

Desde já agradecemos pela sua colaboração.

Atenciosamente,

Fernanda Costa Batista

(Pesquisadora responsável pela pesquisa)

CONSENTIMENTO DA PARTICIPAÇÃO DA PESSOA COMO SUJEITO

$\mathrm{Eu}$,

, CPF (opcional): li/fui informado e estou de acordo com as informações acima referidas.

Informo que recebi uma via deste documento com todas as páginas rubricadas e assinadas por mim e pelo Pesquisador.

Autorizo o pesquisador acessar o meu "Prontuário do Paciente" para a coleta dos dados/informações necessárias para a realização desta pesquisa.

Local e data:

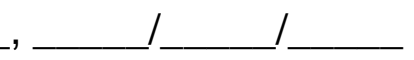

Nome e assinatura do participante da pesquisa:

Assinatura datilográfica (polegar direito ou esquerdo) 


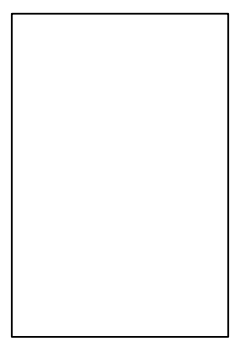

Presenciamos a solicitação de consentimento, esclarecimentos sobre a pesquisa e aceite do participante. Testemunhas (não ligadas à equipe de pesquisadores)

Nome:

Assinatura:

Local e data: Local e data: 1

APENDICE B

FICHA DE INFORMAÇÕES COMPLEMENTARES

IDENTIFICAÇÃO:

Nome:

Data De Nascimento: Idade: Sexo: ( )Fem ( ) Masc

Número do prontuário:

Escolaridade:
( ) Analfabeto
( ) Ensino Médio Completo
( ) Ensino Fundamental Incompleto
( ) Ensino Superior Incompleto
( ) Ensino Fundamental Completo
( ) Ensino Superior Incompleto
( ) Ensino Médio Incompleto
( ) Pós-graduação/MBA/mestrado

Estado Civil:

( ) casado(a) ( ) solteiro(a) ( ) divorciado ( ) União estável

( ) outro:

Profissão:

Telefone: 
Artigo

Atenção à saúde

E-mail:

DADOS ANTROPOMÉTRICOS

Peso Pré-Operatório $(\mathrm{Kg})$ :

Peso Atual $(\mathrm{Kg})$ :

Altura (m):

IMC Pré-Operatório:

IMC Atual:

\section{DADOS DA CIRURGIA}

Técnica Cirúrgica:

Tempo de internação no Pós-Operatório em dias:

Tempo totalde Pós-Operatório em dias:

COMORBIDADES:

Presença de comorbidades antes da cirurgia:

Presença de comorbidades após a cirurgia:

COMPLICAÇÕES APÓS A CIRURGIA

Imediatas:

Tardias: 


\section{ANEXO I \\ FICHA DE PONTUAÇÃO DAS COMORBIDADES E COMPLICAÇÕES}

Perda do excesso de peso e comorbidades

\begin{tabular}{|c|c|c|c|c|c|}
\hline $\begin{array}{c}\text { Perda de } \\
\text { Excesso de } \\
\text { Peso }\end{array}$ & $\begin{array}{c}\text { Ganho } \\
\text { de peso } \\
(-1)\end{array}$ & $0-24(0)$ & $25-49(1)$ & $50-74(2)$ & $75-100(3)$ \\
\hline $\begin{array}{c}\text { Condições } \\
\text { médicas }\end{array}$ & $\begin{array}{c}\text { Agravada } \\
(-1)\end{array}$ & $\begin{array}{c}\text { Inalterado } \\
(0)\end{array}$ & $\begin{array}{c}\text { Melhorado } \\
(1)\end{array}$ & $\begin{array}{c}\text { Uma } \\
\text { importante } \\
\text { resolvida, } \\
\text { outras } \\
\text { melhoraram } \\
(2)\end{array}$ & $\begin{array}{c}\text { Todos os } \\
\text { principais } \\
\text { resolvidas } \\
\text { outros } \\
\text { melhoraram } \\
(3)\end{array}$ \\
\hline
\end{tabular}

Complicações:

Menor: Dedução 0.2 ponto

Maior: Deduzir 1 ponto

Reoperação: deduzir 1 ponto

Complicações precoces e tardias após a cirurgia bariátrica.

Diretrizes para usar o BAROS. Uma grande complicação é definida por um evento que resulta em uma estadia hospitalar $>\mathrm{Ou}=\mathrm{a} 7$ dias.

\section{Complicações cirúrgicas}
A. Principal ( Grave)

1. Imediata: vazamento Gl com peritonite ou abscesso grave; infecção da ferida, deiscência; Hemorragia intraperitoneal; sangramento gastrointestinal que requer transfusão; Lesão do baço que requer esplenectomia ou outra lesão grave do órgão; íleo grave; Obstrução intestinal, síndrome do volvulus / ciclo fechado; dilatação gástrica aguda.

2. Tardia: Doença da úlcera péptica complicada; Colelitíase, hérnia incisional, ruptura da linha de grampeamento; fístula gastro-gástrica ou erosão da banda que requer uma reoperação; Reoperação por deficiência protéica severa ou outros problemas nutricionais.

B. Menor (Moderado) 
1. Imediata: seroma; infecção menor de ferida/ pele; edema de estoma.

2. Tardia: Estenose do estoma; desequilíbrio eletrolítico; vômito persistente ou náuseas; Esofagite,; esófago de Barret; úlcera marginal; úlcera péptica.

\section{Complicações médicas}

A. Principal (Grave)

1. Imediata: Pulmonares (Pneumonia, atelectasias graves, insuficiência respiratória, edema pulmonar Embolia pulmonar, síndrome de dificuldade respiratória do adulto CARDS); Cardiovasculares (Infarto do miocárdio, insuficiência cardíaca congestiva, acidente vascular cerebral); Renal (Insuficiência renal aguda); Psiquiátrico (Depressão pós-operatória severa; psicose).

2. Tardia: Hepático (Falencia hepática, cirrose); Psiquiátrico (Anorexia nervosa, bulimia, depressão maior

B. Menor (Moderado)

1. Imediata: Atelectasia, infecção do trato urinário, trombose venosa profunda sem embolismo; Desequilíbrio eletrolítico, vômito, esofagite

2. Tardia: Anemia, deficiência metabólica (vitaminas / minerais / proteínas), perda de cabelo

\section{Pontuação dos resultados:}

INSUFICIENTE: 1 ponto ou menos

MODERADA: $>1$ a 3 pontos

$\mathrm{BOA}:>3$ a 5 pontos

MUITO BOA: $>5$ a 7 pontos

EXCELENTE: $>7$ a 9 ponto 


\section{ANEXO II}

\section{QUESTIONÁRIO BAROS (BARIATRIC ANALYSIS AND REPORTING OUTCOME SYSTEM)}

1)Após a operação para a obesidade eu sinto que de uma maneira geral, fiquei:
a)Muito pior $(-1,0)$
b)Pior $(-0,5)$
c) Igual (0)
d)Melhor $(+0,5)$
e)Muito melhor $(+1,0)$

2)Minhas atividades físicas:
a)Diminuíram muito $(-0,5)$
b)Diminuíram $(-0,25)$
c)Estão na mesma (0)
d)Aumentaram $(+0,25)$
e)Aumentaram muito $(+0,5)$

3)Estou frequentando reuniões sociais e familiares:
a)Muito menos $(-0,5)$
b)Menos $(0,25)$
c)Sem mudanças (0)
d)Mais $(+0,25)$
e)Muito mais $(+0,5)$

4)Minha capacidade de trabalho está:
a)Muito reduzida $(-0,5)$
b)Reduzida $(-0,25)$
c) Inalterada (0)
d)Melhorada $(+0,25)$
e)Muito melhorada $(+0,5)$ 
Artigo

Atenção à saúde

5)Meu interesse por sexo tornou-se:
a)Muito menor $(-0,5)$
b)Menor $(-0,25)$
c) Igual (0)
d)Maior $(+0,25)$
e)Muito maior $(+0,5)$ 